\title{
Excess Li ions in a small graphite cluster
}

\author{
M. Nakadaira, R. Saito, and T. Kimura \\ Department of Electronics Engineering, University of Electro-Communications, Chofugaoka, Chofu, \\ 182 Tokyo, Japan \\ G. Dresselhaus \\ Francis Bitter Magnet Laboratory, Massachusetts Institute of Technology, Cambridge, \\ Massachusetts 02139 \\ M. S. Dresselhaus \\ Department of Electrical Engineering and Computer Science and Department of Physics, \\ Massachusetts Institute of Technology, Cambridge, Massachusetts 02139
}

(Received 28 October 1996; accepted 23 December 1996)

\begin{abstract}
We calculate the optimized geometry and the corresponding electronic structure of $\mathrm{Li}$ ions doped in a small graphite cluster with dangling bonds or hydrogen terminations at the edge surrounding the cluster. The calculations imply both covalent and ionic bonds of Li ions to carbon atoms, which may be relevant to explaining the broad signal of the ${ }^{7} \mathrm{Li}$ NMR Knight shift spectra. Li intercalation, in particular, is possible even at the hydrogen-terminated edges. Because of the finite size effect of the cluster, the ionicity of intercalated $\mathrm{Li}$ ions has a large distribution of values, ranging from positive values close to that in graphite intercalation compounds to even slightly negative values, depending on the bonding geometry. We propose that the cluster edge surface plays a special role in accommodating excess $\mathrm{Li}$ ions in the disordered graphite system.
\end{abstract}

\section{INTRODUCTION}

Because of advances in computer technology it has recently become possible to calculate the electronic structure for much larger arrays consisting of hundreds (or thousands) atoms in a non-periodic system, thus allowing exploration of the optimized stable geometry and electronic structure of an atomic cluster or the surface of materials. In the small graphite clusters that are pyrolytically synthesized from hydrocarbons, such as poly-paraphenylene (PPP) $)^{1,2}$ and phenol-formaldehyde (polyacen semiconductors, PAS), ${ }^{3-5}$ the graphite cluster can accept three times as many $\mathrm{Li}$ ions $\left(\mathrm{LiC}_{2}\right)$ as the first stage $\mathrm{Li}$ intercalated $\left(\mathrm{LiC}_{6}\right)$ graphite intercalation compound (GIC). In GIC's, $\mathrm{LiC}_{6}$ is the compound with the greatest concentration of intercalate atoms that can be introduced between graphite layers under ambient pressures, forming a $\sqrt{3} \times \sqrt{3}$ structure of $\mathrm{Li}$ ions on a honeycomb lattice, as in first stage Li-GIC's. ${ }^{6}$ The fact that the disordered graphite can absorb three times as many $\mathrm{Li}$ ions as GIC's is striking. This property can be applied, in principle, to produce new lightweight secondary batteries for electric cars and solar cell applications. ${ }^{1,4}$

Recently, fullerenes, a newly discovered form of carbon, have aroused a great deal of interest in many fields of science. ${ }^{7,8}$ Among the many forms of fullerenes, the most stable is the $\mathrm{C}_{60}$ molecule that can be doped exohedrally with up to $12 \mathrm{Li}$ atoms, ${ }^{9}$ to achieve a cluster with the stoichiometry $\mathrm{Li}_{12} \mathrm{C}_{60}$, which means that more $\mathrm{Li}$ ions per carbon atom can be adsorbed on the $\mathrm{Li}_{12} \mathrm{C}_{60}$ cluster than in $\mathrm{LiC}_{6}$, the first stage Li-GIC. ${ }^{9}$ Although the electronic structures of these carbon isomers, such as graphite (semi-metal), PAS, and $\mathrm{C}_{60}$ (semiconductors), are different, it is clear that the ionic radius of $\mathrm{Li}^{+}$ ions $(0.94 \AA$ in the alkali-halide structure and $0.68 \AA$ in the closed-packed metallic structure $)^{10}$ determines the maximum density possible for $\mathrm{Li}$ ions, as is discussed in the physics of GIC's. ${ }^{6}$ Thus, the positive curvature of the $\mathrm{C}_{60}$ molecular surface and the enhanced reactivity of the edge of PPP or PAS graphite clusters might play a key role in making more space available for $\mathrm{Li}$ ions, and therefore may be relevant to the absorption of excess $\mathrm{Li}$ ions by these clusters.

In a recent ${ }^{7} \mathrm{Li} \mathrm{NMR}$ experiment, Sato et al. have shown that there are two kinds of $\mathrm{Li}$ sites which give different Knight shift values. ${ }^{2}$ This clearly shows the existence of different types of chemical bonding between $\mathrm{Li}$ ions with themselves or with carbon atoms in which the electronic structure associated with the edges should be different from that with the bulk. In order to explain the ${ }^{7} \mathrm{Li}$ NMR spectra, the authors tentatively proposed the existence of molecular $\mathrm{Li}_{2}$ states in $\mathrm{LiC}_{6}$ in which all hexagonal centers of the honeycomb lattice are occupied by $\mathrm{Li}$ atoms to give the $\mathrm{LiC}_{2}$ stoichiometry. ${ }^{2}$ However, a large amount of energy should be needed to put $\mathrm{Li}$ atoms in a plane, and even in this case, some three-dimensional structure would be expected to occur. Such issues have motivated the present calculation.

In this paper, we focus on the reason why a small graphite cluster can accept more $\mathrm{Li}$ ions than bulk graphite. For this purpose we calculate the optimized 
geometry and electronic structure for placing Li ions on a graphite surface, using a semi-empirical method. In Sec. II we explain the method that we used and the ideas why we select the present clusters as models for the calculation. In Sec. III we present the calculated results, and in Sec. IV a discussion and summary are given.

\section{METHOD AND IDEAS}

The calculation method that we used is the semi-empirical quantum mechanics calculation library, MOPAC93 ${ }^{11}$ with the lattice optimization option of the parametric method 3 (PM3). ${ }^{12}$ In MOPAC93 the matrix elements of the Hamiltonian are empirically given as a function of the distance between two atoms, including the two-electron integrals of the Hartree and exchange interactions. The electronic structure calculation of a cluster is performed within the unrestricted Hartree-Fock calculation only for the valence electrons. The dangling bond and ionicity can be well described within this approximation as shown below. As for the core electrons we consider the effective core potential for calculating the forces between two atoms, whose model function is optimized so as to reproduce the well-known behavior of many chemical molecules in the PM3 model functions. ${ }^{13} \mathrm{~A}$ total energy is given by the sum of electronic energy of valence electrons and the core repulsion energy. Since the repulsion energy between two Li ions, which are located above the center of a hexagonal ring in the honeycomb lattice, is relatively large compared with the electrostatic potential of the host honeycomb lattice, $\mathrm{Li}$ atoms can easily move on graphite surfaces. Thus many iterations of the calculation are necessary to obtain the optimized geometry for $\mathrm{Li}$ ions on a graphite surface. In order to get a general idea about the motion of $\mathrm{Li}$ ions on graphite surfaces, we tried many geometries as initial conditions. In this paper, we show only typical results for $\mathrm{Li}$ ions forming different types of chemical bonds. A semi-empirical calculation is thus suitable for the present purpose. We have calculated the electronic structure for $\mathrm{C}_{96} \mathrm{Li}$ clusters using a workstation, and for $\mathrm{C}_{96} \mathrm{H}_{y} \mathrm{Li}_{x}$ clusters using a VPP-500 supercomputer in the computer center of the Institute for Solid State Physics, Tokyo University. It sometimes requires several hours to obtain an optimized geometry, if we calculate the electronic structure for a cluster with several $\mathrm{Li}$ ions using a single VPP-500 processor.

For simplicity in the calculation, the bond angle between carbon atoms and hydrogen atoms is fixed, and the bond lengths of carbon atoms and hydrogen atoms are optimized. Furthermore, the position of a $\mathrm{Li}$ atom is fully optimized geometrically in the calculation. In fact, even if we relaxed the carbon atom locations near the $\mathrm{Li}$ atoms, the optimized carbon atoms would shift from the solid state graphite values by less than $0.006 \AA$. Calculation of this relaxation is therefore not essential for the present purpose.

Since pyrolytic carbon materials are normally generated from hydrocarbon precursors, hydrogen atoms commonly terminate the edge of the graphite cluster under low heat treatment temperature $\left(T_{\mathrm{HT}} \sim 700{ }^{\circ} \mathrm{C}\right)$ conditions. $^{2}$ However, the hydrogen atoms begin to desorb from the edge at the lower $T_{\mathrm{HT}}$ between $600{ }^{\circ} \mathrm{C}$ and $700{ }^{\circ} \mathrm{C}$, so that dangling bonds appear at the edge. In this paper we consider edge bonding, both with or without hydrogen termination, to examine the role of hydrogen in bonding $\mathrm{Li}$ to carbon. We found in the present calculation that $\mathrm{Li}$ ions can be intercalated even at edges terminated by hydrogen.

In this calculation, we consider a hexagonal graphite cluster, $\mathrm{C}_{96}$, as is shown in Fig. 1(a), into which we introduce up to seven Li ions. With regard to the host geometry, we consider, too, an all-hydrogen-terminated cluster $\mathrm{C}_{96} \mathrm{H}_{24} \mathrm{Li}_{x}(x=1 \ldots 7)$, as is shown in Fig. 1(b), and partially hydrogen-terminated clusters $\mathrm{C}_{96} \mathrm{H}_{20} \mathrm{Li}_{x}$ and $\mathrm{C}_{96} \mathrm{H}_{16} \mathrm{Li}_{x}$. In Figs. 1(a) and 1(b), where $\mathrm{C}_{96}$ and $\mathrm{C}_{96} \mathrm{H}_{24}$ are shown, respectively, a $\mathrm{Li}$ ion is placed over the center of the honeycomb lattice at a distance of $1.9 \AA$ from the graphite plane. In Fig. 1 we also show the six inequivalent $\mathrm{Li}$ sites on the graphite cluster which are denoted by $A$ to $F$. The diameter of these clusters is about $20 \AA$, whose size is close to the clusters observed in PPP-derived graphite $(20-30 \AA) .{ }^{14}$

An important feature of the present problem is the shape of the edges. In graphite there are two different types of cut ends that can be made of $s p^{2}$ covalent bonds. These, respectively, show the cis and trans type geometry observed in polyacetylene, $(\mathrm{CH})_{x}$, and are called armchair and zigzag edges in carbon nanotubes, ${ }^{15}$ respectively. In Fig. 1, we show the armchair and zigzag edges as solid and open bonds at the surface of the cluster, respectively. It is clear from Fig. 1 that we find the armchair and zigzag edges at the corners and at the sides of the cluster, respectively. The number of corresponding carbon atoms for armchair and zigzag edges is the same, 12 in the present $\mathrm{C}_{96}$ cluster. The electronic structures of armchair and zigzag edges are, however, very different, since nearest-neighbor carbon atoms at the cut ends exist in the armchair edge, but not in the zigzag edge. Thus the edge states for the zigzag edge are almost non-bonding, while the corresponding edge states of the armchair edge have an energy gap between bonding and antibonding orbitals. ${ }^{16-18}$ This situation reflects charge transfer from the $\mathrm{Li}$ ion to the graphite cluster.

When Li ions are near the zigzag edges, the charge transfer will occur from $\mathrm{Li}$ ions to the edge states, because the unoccupied, non-bonding edge states of the zigzag edges are near the Fermi energy, and have a lower energy than the $2 s$ states of a $\mathrm{Li}$ atom. In the case of the 

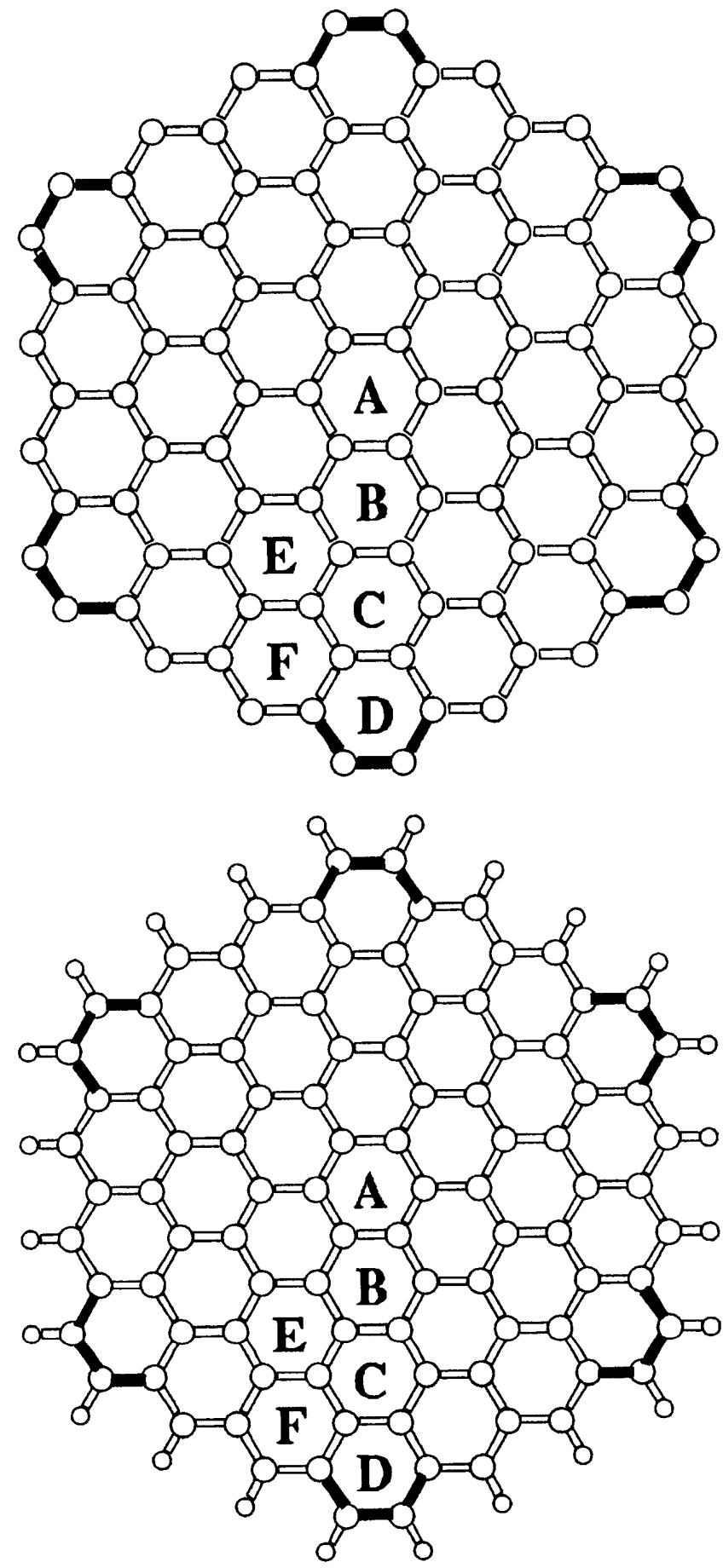

FIG. 1. (a) A graphite cluster, $\mathrm{C}_{96}$ and (b) a hydrogen terminated graphite cluster $\mathrm{C}_{96} \mathrm{H}_{24}$. The six inequivalent positions of the center of a hexagon in the $\mathrm{C}_{96}$ cluster are labeled from $A$ to $F$. On the edge of clusters, armchair and zigzag edges exist at the corners and at the sides of the cluster, denoted as solid and open bonds, respectively.

armchair edges, however, the unoccupied, non-bonding edge states do not always have a lower energy than the $2 s$ states of a Li atom, since there is an energy gap centered about the Fermi energy between the bonding and antibonding armchair edge states. If the one-electron energy of unoccupied, armchair edge states is higher than that of the Li $2 s$ states, then the charge transfer from the $\mathrm{Li}$ atoms occurs not to the edge states but rather to bulk graphite unoccupied states.

This situation will clearly occur in the $\sigma$ orbital at the hydrogen-terminated edges, in which there are tight binding $\sigma$-bonds between the dangling bonds with $\mathrm{H} 1 s$ states. In this case the unoccupied $\sigma$ states should have a higher energy than the Li $2 s$ levels, and thus no charge transfer occurs from the $\mathrm{Li} 2 s$ state to the unoccupied $\sigma$ states. In this sense, the $\mathrm{H}-\mathrm{C} \sigma$ bond cannot be simply substituted by a $\mathrm{Li}-\mathrm{C} \sigma$ bond. However, since bulk unoccupied $\pi$ states near the Fermi energy exist, the intercalation of Li ions near H-terminated edges is possible, as shown below.

Before showing the calculated results, let us briefly mention the case of multiple Li ions. Because of the existence of $\mathrm{Li}-\mathrm{Li}$ metallic bonds and the weak interaction between graphite and $\mathrm{Li}$ ions, the $\mathrm{Li} 2 s$ states form $2 s$ energy bands. If the number of $\mathrm{Li}$ atoms is finite, then finite molecular orbitals (MO's) consisting of $\mathrm{Li} 2 s$ states appear within the energy band width of the bulk Li $2 s$ band. Thus the relative energies of Li MO's with respect to the edge state energies or $E_{F}$ may depend on the molecular orbital, and some Li MO's are below $E_{F}$. Since the amplitude of the wave function of the occupied MO's is not always the same for all the Li ion sites, the Li ionicity is distributed over a range of value depending on the specific site, and this effect may be relevant to the broad signal observed for the ${ }^{7} \mathrm{Li}$ Knight shift. ${ }^{2}$

\section{CALCULATED RESULTS AND DISCUSSION}

\section{A. Single $\mathrm{Li}$ ion on a graphite cluster $\mathrm{LiC}_{96}$}

First we put a single $\mathrm{Li}$ ion on the graphite cluster $\mathrm{C}_{96}$ at $1.9 \AA$ from the graphite surface above the center of a hexagonal ring for the initial condition. In the present calculation we consider the six possible inequivalent initial sites ( $A$ to $F$ in Fig. 1) for a single Li ion. When we put the $\mathrm{Li}$ ion at the center of the graphite cluster ( $A$ site), the $\mathrm{Li}$ ion is metastable under the initial conditions, and the equilibrium distance between the $\mathrm{Li}$ and the six equivalent nearest carbon atoms is found to be $2.66 \AA$. The ionicity of the $\mathrm{Li}$ ion is $+0.7 e$ at the optimized $A$ site. Thus we can say that the $\mathrm{Li}$ atom at the optimized $A$ site is ionic, as is observed in GIC's.

When we initially locate the $\mathrm{Li}$ ions at $B$ to $F$ sites, all the Li ions subsequently move to the edges and make a covalent $(\sigma)$ bond with two dangling carbon bonds, as shown in Fig. 2. The covalently-bonded Li ioncarbon distance at the edges is $2.1 \AA$, as shown in the expanded section of Fig. 2. When compared to the total energy of $\mathrm{LiC}_{96}$, the total energy of the covalent $\mathrm{Li}$ ion 


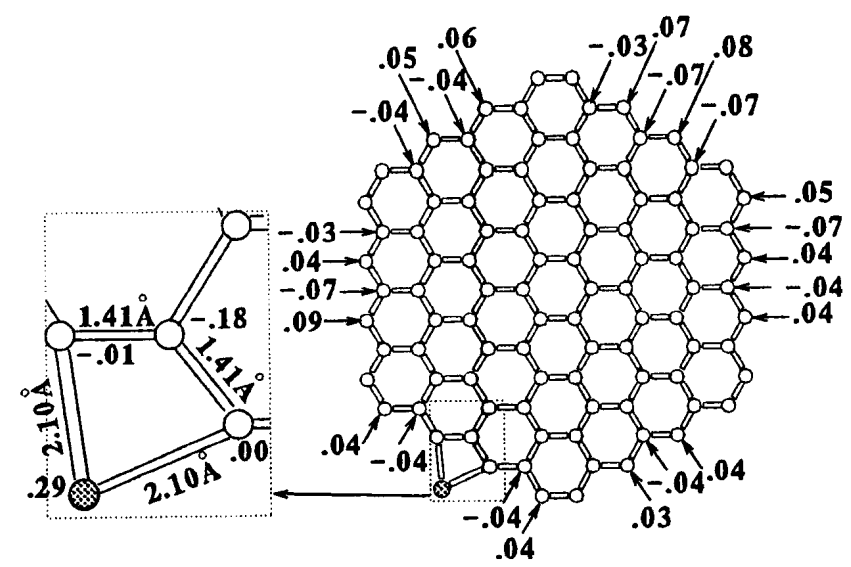

FIG. 2. An optimized Li geometry in the $\mathrm{C}_{96}$ cluster in which a $\mathrm{Li}^{+0.29}$ ion is mostly covalently bonded with the two nearest-neighbor carbon atoms. The charge transfer from the $\mathrm{Li}$ ion to the graphite cluster is located at the edge region. However, the ionicities of the two nearest-neighbor carbon atoms are almost zero. We show the ionicities of atoms whose absolute values are more than $0.03 e$.

is about $8 \mathrm{eV}$ lower than that of the ionic $\mathrm{Li}$ ion at the $A$ site. Thus the symmetric optimized geometry of ionic $\mathrm{Li}$ corresponds to a local minimum of the total energy.

In Fig. 2, an optimized Li geometry in the $\mathrm{C}_{96}$ cluster is shown in which the $\mathrm{Li}^{+0.29}$ ion is mostly covalently bonded with the two nearest-neighbor carbon atoms. The charge that is transferred from the $\mathrm{Li}$ ions to the graphite cluster is located at the edge region; however, the ionicity of the two nearest-neighbor carbon atoms is almost zero. According to the tight binding calculation, the zigzag edge states of the neutral graphite surface consist of carbon atoms with dangling bonds. However, the charge transfer from Li ions is not to the zigzag edge states but to some other surface states of graphite. These facts clearly show that there is a hybridization of $\mathrm{Li} 2 s$ states with the carbon zigzag edge states to form bonding states. This accounts for the low ionicity of the $\mathrm{Li}$ ions, $+0.29 e$, in this case. The electron transferred from the $\mathrm{Li}$ ions is mainly located at the second row surface atom, as shown in the enlarged section of Fig. 2, and the value of the charge is $-0.18 e$. The remaining charge is delocalized in the first and second row surface carbon atoms over the cluster, as shown in Fig. 2. It should be mentioned that the charge distribtuion over the cluster in Fig. 2 is not symmetric. Since the cluster has an odd number of electrons, we used the unrestricted Hartree-Fock calculation in which the wave functions for up and down spins are calculated independently, so that the self-consistent charge or spin does not always have the symmetry of the cluster.

\section{B. Single Li ion on a hydrogen-terminated graphite cluster $\mathrm{LiC}_{96} \mathrm{H}_{24}$}

Next we calculate the $\mathrm{Li}$ ion optimization for a hydrogen-terminated cluster, $\mathrm{LiH}_{24} \mathrm{C}_{96}$. Our calculations show that when hydrogen atoms terminate the dangling bonds, $\mathrm{Li}$ ions cannot be substituted for $\mathrm{H}$ atoms. When we select initial conditions $A$ to $D$, the optimized $\mathrm{Li}$ position is close to the initial site. The ionicity of $\mathrm{Li}$ is $0.7 e$ which is close to the previous ionic case. As for the other initial conditions $E$ to $F$, the $\mathrm{Li}$ ion moves to the edge between two adjacent $\mathrm{H}$ atoms in which the nearest $\mathrm{Li}-\mathrm{C}$ distance is $2.3 \AA$ and the $\mathrm{Li}$ ion is $1.83 \AA$ from the graphite plane, as shown in Fig. 3.

The ionicity of each $\mathrm{Li}$ ion in this case is $\sim 0.6 e$, and this is thus another possible case of ionic bonding or intercalation, since the ionicity of the $24 \mathrm{H}$ atoms does not change much from an ionicity of $+0.10 e$. The remaining electrons are in unoccupied edge states of the $\pi$ bands, as shown in Fig. 3 where the amplitude is large in the first row of the surface of the graphite cluster near the $\mathrm{Li}$ ion. It is interesting to note that the ionicity of $\mathrm{H}$ atoms near the $\mathrm{Li}$ ion becomes even more positive $(0.12 e)$ than the other $\mathrm{H}$ atoms $(0.10 e)$. The total energy of the $\mathrm{Li}$ ion intercalated at hydrogen terminated edges is $2.4 \mathrm{eV}$ more stable than the $\mathrm{Li}$ ions ( $A$ to $D$ ) in the inner region.

In Figs. 4(a) and 4(b), we compare the charge density map of the valence electrons of the two $\mathrm{Li}$ ions of Figs. 2 and 3, that are in a plane which is parallel to a graphene sheet $(z=$ constant $)$ at the Li positions $(z=0$ and $z=1.83 \AA$ ), respectively. In Fig. 4(a), there is almost no charge density at the Li position, though the ionicity of the Li ion is +0.29 . The place that has a large charge density is at the dangling bonds of carbon and on the graphene lattice denoted by thick solid lines. From the figure we see that the electronic charges transferred from the Li ion mainly reside in the dangling bond states which consist of $\mathrm{C} 2 p_{x}$ and $2 p_{y}$ orbitals. There is almost

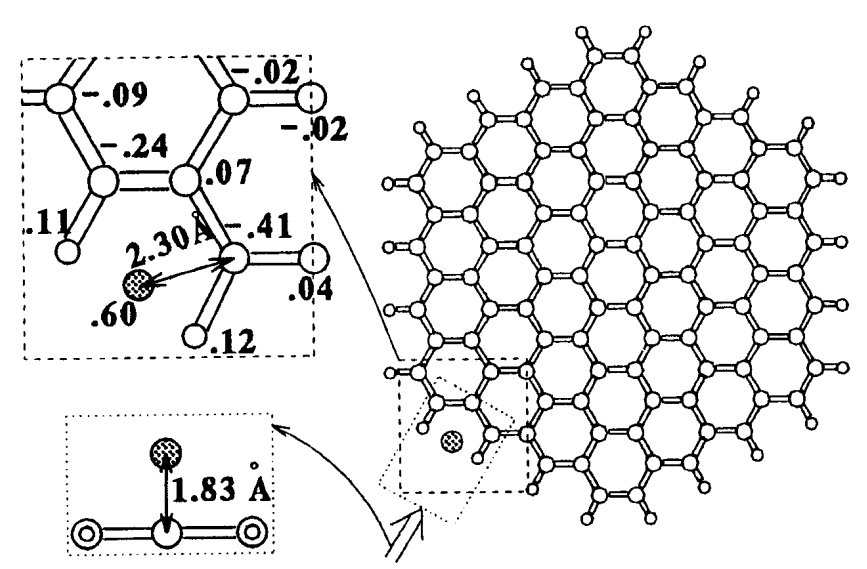

FIG. 3. An optimized Li geometry in the $\mathrm{LiC}_{96} \mathrm{H}_{24}$ cluster when the initial position of the $\mathrm{Li}$ ion is from $E$ to $F$ in Fig. 1 . Li intercalation at hydrogen-terminated edges is possible. In the insets we show the $\mathrm{Li}$ position and the ionicities from the top view and from the side view of an enlargement of the area near the Li ion. We show the ionicities of atoms whose absolute values are more than $0.03 e$. 


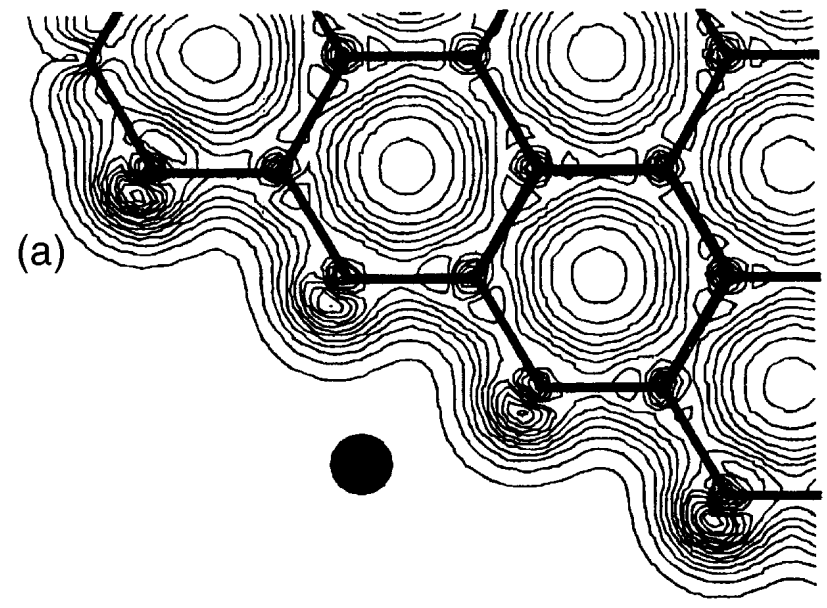

(b)

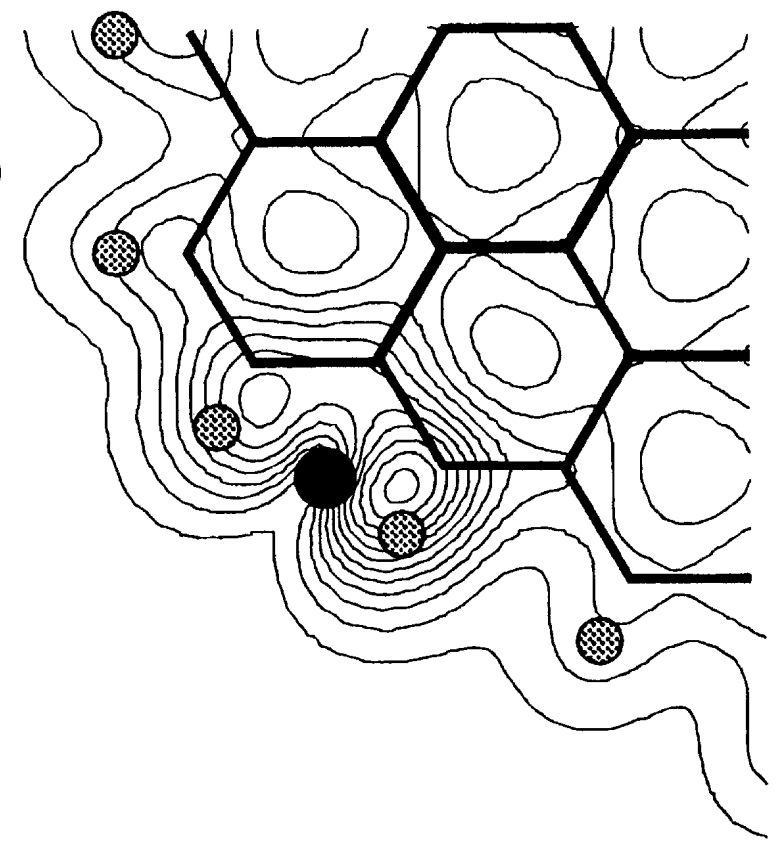

FIG. 4. Charge density map of the valence electrons near the Li ions for (a) Fig. 2 and (b) Fig. 3. The map is taken in the plane parallel to the graphene lattice, which is denoted by thick solid lines and the Li position is denoted by a solid circle. Shaded circles denote the positions of hydrogen atoms

no bonding with $\mathrm{C} 2 p_{z}$ orbitals because of symmetry. Not only because of the large overlap of the Li $2 s$ orbital with the dangling bond states of the carbon atoms, but also because of the hybridization of the Li $2 s$ orbital with $\mathrm{Li} 2 p_{x}$ and $2 p_{y}$ orbitals, the small ionicity of the $\mathrm{Li}$ arises from the bond contribution of electronic charge of the ion. Since the lithium electron is not on the atom but is rather in the $\mathrm{Li}-\mathrm{C}$ bonds predominantly, we can say that this Li ion is covalently bonded.

On the other hand in Fig. 4(b), the charge density is localized near carbon atoms closest to the $\mathrm{Li}$ atoms. Since the position of the $\mathrm{Li}$ in this case is $z=1.83 \AA$, the $\mathrm{Li}$ ions are mainly coupled with the $\mathrm{C} 2 p_{z}$ orbitals. The Li $2 s$ electron is thus transferred to the $2 p_{z}$ orbital of the nearest carbon atom. It is known that the $\pi$ orbitals at the zigzag edge atoms are localized in the edge region whose energy is near the Fermi energy. Further the Li $2 p_{z}$ component in the occupied molecular orbitals is small since the electron-electron repulsive interaction makes the energy of the Li $2 p_{z}$ level to lie higher than that in the case of the neutral atom. Thus the Li components of the charge are relatively smaller than for the hybridized case in Fig. 4(a). In fact the ionicity, +0.60 , for Fig. 4(b) is larger than for the case of Fig. 4(a). Thus the $\mathrm{Li}$ atom in Fig. 4(b) is relatively ionic.

\section{Li ions on the clusters with dangling bonds: $\mathrm{Li}_{7} \mathrm{C}_{96}$}

The local stability at the $A$ site observed in $\mathrm{Li}_{1} \mathrm{C}_{96}$ is weak compared with the $\mathrm{Li}-\mathrm{Li}$ interaction. Thus, when we start from seven $\mathrm{Li}$ ions in a $\sqrt{3} \times \sqrt{3}$ structure consisting of one $A$ site and six $E$ sites, the geometrical optimization procedure moves all seven of the $\mathrm{Li}$ ions out to the edge. In Fig. 5 we show an example of the optimized geometry of seven $\mathrm{Li}$ ions with their own ionicities and also the ionicities of the carbon atoms to which most of the electron charge is transferred from the $\mathrm{Li}$ ions. Here we see that six $\mathrm{Li}$ ions (denoted as $A 1-A 3$ and $Z 1-Z 3$ ) are located at the edges of the cluster within the graphite plane to form $\sigma$ bonds with the adjacent carbon atoms. The remaining $\mathrm{Li}$, denoted by $N$, is a neutral species and lies far $(10.6 \AA)$ from the plane of the graphite. The cluster in Fig. 5, as seen from a top view, thus indicates that there is no bonding between this $\mathrm{Li}$ atom and the graphite layer, so that the $\mathrm{Li}$ atom can easily leave the cluster. Such a situation sometimes occurs in the calculations because the $\mathrm{Li}-\mathrm{Li}$ repulsive interaction provides enough energy for a $\mathrm{Li}$ ion to leave the cluster. The optimized geometry is a geometry in a local minimum of the total energy. Thus the final geometry may change by a small change of the initial geometry. Although this situation reflects a real system, it is not an essential point. An important fact is that all $\mathrm{Li}$ atoms move to the edge of the cluster in this case.

Among the six $\mathrm{Li}$ ions, three $\mathrm{Li}$ ions, $A 1-A 3$, are connected to the armchair edges at the corners of the cluster, and the three other $\mathrm{Li}$ ions, $Z 1-Z 3$, are connected to the zigzag edges. Since the numbers of carbon atoms at armchair and zigzag edges are equal, it seems reasonable that we find an equal number of $\mathrm{Li}$ ions at armchair and zigzag edges. One $\mathrm{Li}$ ion at the armchair edge $(A 1)$ forms a triangular ring with the nearest two carbon atoms. The ionicity of the $A 1 \mathrm{Li}$ is $+0.57 e$ (see Fig. 5) and the transferred charge to the carbon atoms is located mainly at the two nearest-neighbor carbon atoms. In this sense, this Li ion is relatively ionic compared with the Li ions at zigzag edges observed in Fig. 2 and 


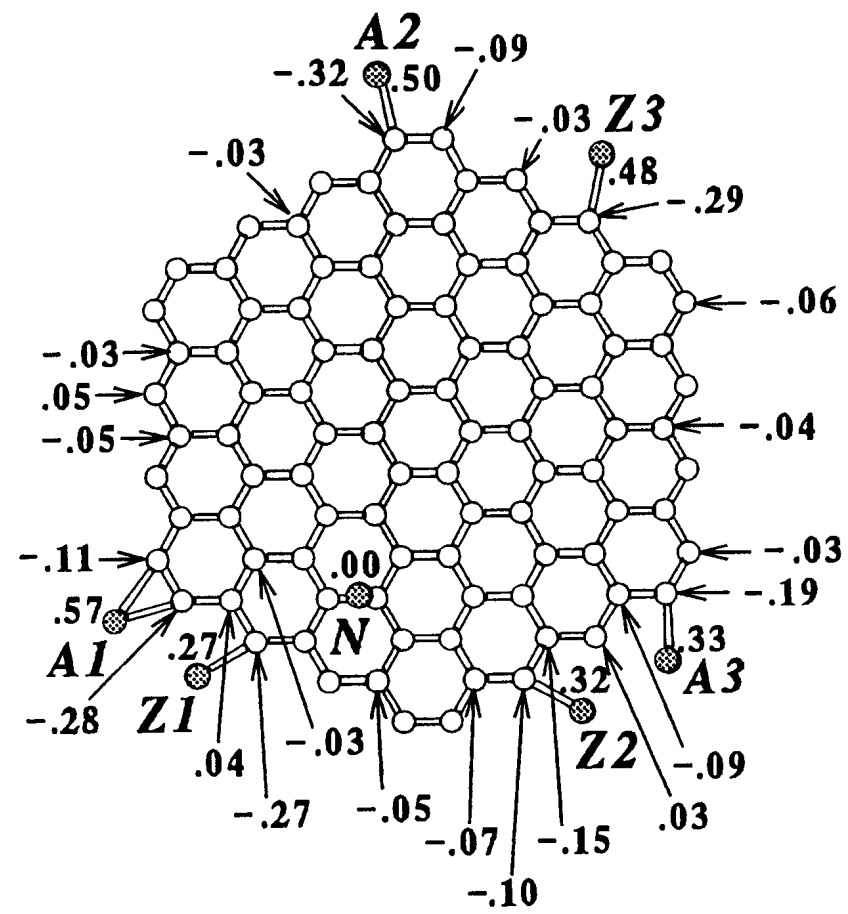

FIG. 5. An optimized Li geometry in the $\mathrm{Li}_{7} \mathrm{C}_{96}$ cluster when the initial positions of the seven Li ions are in the $\sqrt{3} \times \sqrt{3}$ structure. The labels $A 1-A 3, Z 1-Z 3$ denote the six planar $\mathrm{Li}$ ions which connect to the armchair edge, and zigzag edge carbon atoms, respectively, while the $N \mathrm{Li}$ ion is separated from the graphite plane by a large distance $(10.6 \AA)$ and thus becomes a neutral atom which is easily released.

5. Actually, when we compare the total energies of the cluster given in Fig. 2 and the $\mathrm{C}_{96} \mathrm{Li}$ cluster in which a $\mathrm{Li}$ ion is put at an $A 1$ site, the latter cluster is $3 \mathrm{eV}$ less stable than the former.

The other two $\mathrm{Li}$ atoms $A 2$ and $A 3$ at armchair edges are optimized in different geometries from $A 1$. The optimized bond angle for $\mathrm{Li}-\mathrm{C}-\mathrm{C}$ depends sensitively on the local geometries of other $\mathrm{Li}$ ions. Thus we can say that the direction of the $\mathrm{Li}-\mathrm{C}$ bonds can be relatively freely taken, though all the $\mathrm{Li}$ ions are located within the graphene plane. In fact, all $\mathrm{Li}$ ions at zigzag edges, $Z 1-Z 3$, are distorted from the symmetric position observed in Fig. 2. Since the computer software, xmol, automatically plots chemical bonds depending on the bond length, we could get only one chemical bond length from $\mathrm{Li}$ ions at zigzag edges. Though transfer charge from the $Z 1$ and $Z 2 \mathrm{Li}$ ions occurs to nearestneighbor carbon atoms, we can say that these $\mathrm{Li}$ ions are covalently bonded with these two carbon atoms. In fact, the ionicity of the $Z 1$ and $Z 2 \mathrm{Li}$ ions is close to 0.30. In this sense the $A 3 \mathrm{Li}$ ion has much covalent character, though the nearest-neighbor carbon atom is at an armchair edge. Further, the ionicity of the $Z 3 \mathrm{Li}$ ion is similar to that of $A 2$, and thus $Z 3$ ion is ionic. The reason why the $Z 3 \mathrm{Li}$ ion is ionic is that the $\mathrm{Li}$ ion is distorted so much from the two $\sigma$-bonds that the covalent bonding observed in Fig. 2 is not formed.

An important fact to note here is that there is no electric charge transfer from $\mathrm{Li}$ ions to the inner carbon atoms of the cluster. Only the first and second row of carbon atoms are negatively charged. In Fig. 5, we show only the ionicity of the carbon atoms whose absolute values are more than $0.03 e$. The charge distribution is determined by the one-electron energy of the unoccupied edge states that are hybridized with Li $2 s$ states.

Another important fact is that the ionicity of $\mathrm{Li}$ atoms at the edges varies widely from $0.25 e$ to $0.60 e$, depending on the geometry of the edge shape and the nonlocal arrangement of $\mathrm{Li}$ ions. This behavior shows why ${ }^{7}$ Li NMR Knight shift studies should exhibit broad spectral lines.

In Fig. 6 partial densities of states of (a) Li $2 p_{x}$ and $2 p_{y}$, (b) $2 s$, (c) $\mathrm{C} 2 p_{z}$, and (d) $\mathrm{C} 2 p_{x}$ and $2 p_{y}$ for the $\mathrm{Li}_{7} \mathrm{C}_{96}$ cluster, are plotted in the unit of states of states/eV/atom in which the energy levels are broadened by multiplying the Gaussian factor with $\Delta E=0.21 \mathrm{eV}$. The highest occupied molecular level is at $-5 \mathrm{eV}$ in the middle of an energy gap at which we can see the peak of Li $2 s$ components. This peak corresponds to the neutral $\mathrm{Li}$ atom which is accidentally optimized at the position far from the graphite plane. However, this atom can be useful for our knowing the energy level position of the neutral $\mathrm{Li} 2 s$ state. Below the energy of $-5 \mathrm{eV}$ we can see small, but the same quantity, of Li $2 p_{x}$ and $2 p_{y}$ and $\mathrm{Li} 2 s$ states in the same order, showing the hybridization of the $\mathrm{Li}$ ions in the occupied states for making a bond with the $\mathrm{C}$ dangling bonds. Because of non-zero DOS of Li states in the occupied states, the ionicity of $\mathrm{Li}$ is not +1 . The $\mathrm{C} 2 p_{x}$ and $2 p_{y}$ orbitals is not generally seen in the energy region near Fermi level in the case of bulk graphite. A reason why we have $2 p_{x}$ and $2 p_{y}$ components are dangling bonds states, which is important to determine the electric structure of Li ionicity.

\section{Hydrogen-terminated edges: $\mathrm{Li}_{7} \mathrm{C}_{96} \mathrm{H}_{24}$}

When we put 7 carbon atoms into the $\sqrt{3} \times \sqrt{3}$ structure of the hydrogen-terminated graphite cluster, $\mathrm{C}_{96} \mathrm{H}_{24}$ as shown in Fig. 1(b), the atomic position of the initial condition is found to be stable, as shown in Fig. 7. We interpret this situation as follows. Although Li ions are $2.4 \mathrm{eV}$ more stable at a hydrogen-terminated bond, as shown in Sec. III. B, the $\mathrm{Li}-\mathrm{Li}$ metallic bond will gain more energy than the bond formed by the intercalation of $\mathrm{Li}$ atoms near hydrogen-terminated carbon atoms. At least the gradient of the potential for $\mathrm{Li}$ ions at the initial position is smaller than for the potential in the honeycomb lattice. Although the optimized geometry looks like the case of GIC's, we found that the ionicity 


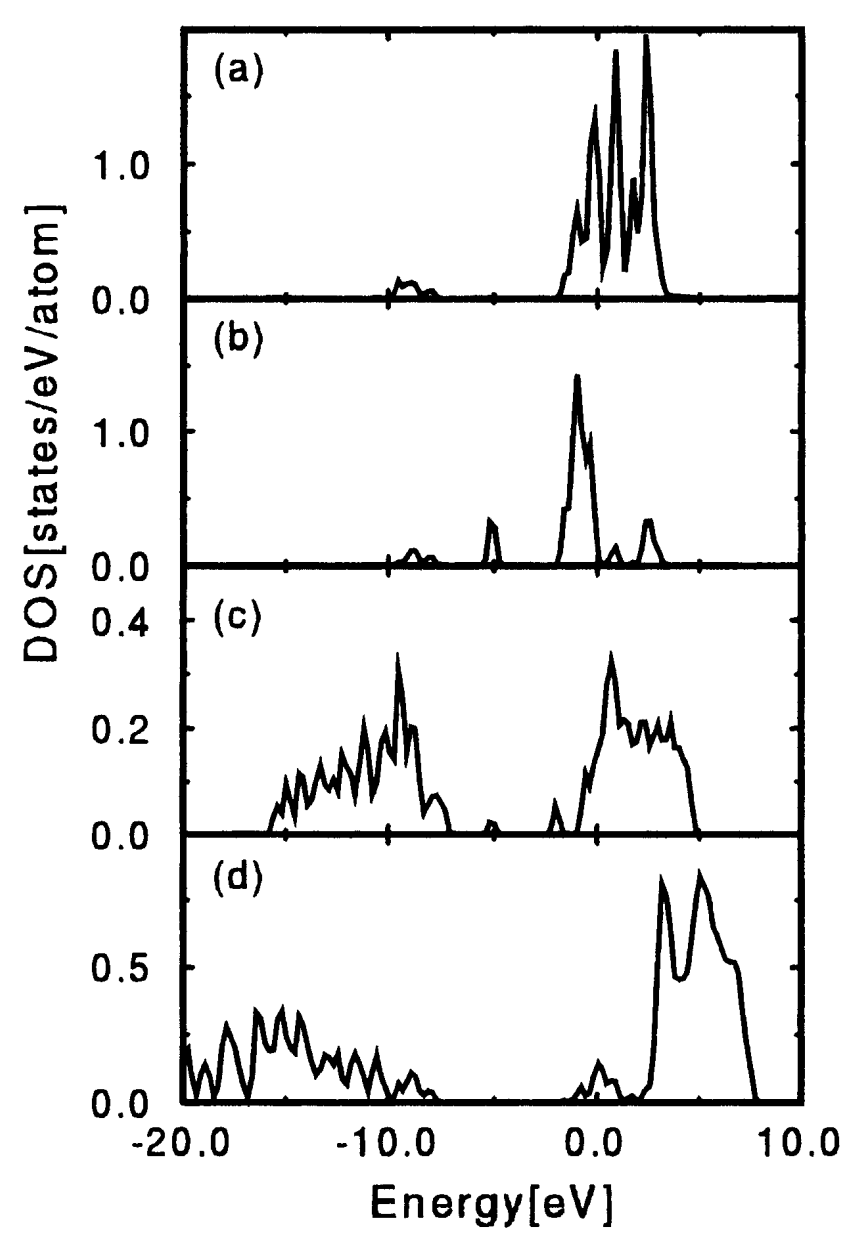

FIG. 6. Partial density of states for (a) Li $2 p_{x}$ and $2 p_{y}$, (b) Li $2 s$, (c) $\mathrm{C} 2 p_{z}$, and (d) C $2 p_{x}$ and $2 p_{y}$ orbitals in the units of states/eV/atom. Here the energy levels are broadened by a Gaussian factor and the direction of $z$ is taken to be perpendicular to the graphene plane.

of three of the Li ions is negative, as shown in Fig. 7. The appearance of a negatively charged $\mathrm{Li}$ ion is not rare in the very small clusters that we calculated here for a variety of initial conditions.

Thus the reason why we sometimes get a negatively charged ion for $\mathrm{Li}$ is due to the expected finite size effect of the graphite cluster. In the $\mathrm{Li}_{7} \mathrm{C}_{96}$ cluster, only two of the seven $\mathrm{Li} 2 s$ electrons in the $\mathrm{Li}_{7} \mathrm{C}_{96}$ cluster move from the seven $\mathrm{Li}$ ions to the graphite cluster. The remaining five $2 s$ electrons are retained by the $\mathrm{Li}$ ions. Thus the average ionicity is only about 0.3 . This is because the $\mathrm{Li}$ $2 s$ orbitals are coupled to each other to form molecular orbitals (MO's). When we examine the coefficients of the atomic wave functions of the molecular orbital, we find seven MO's which mainly consist of seven Li $2 s$ atomic orbitals. Since the $\mathrm{Li}_{7} \mathrm{C}_{96}$ cluster has $C_{6 v}$ point group symmetry initially, the seven Li MO's belong to the following irreducible representations of $C_{6 \nu}: 2 A_{1}$, $B_{1}, E_{1}$, and $E_{2}$. Here $B_{1}$ is the irreducible representation which is invariant under the $\sigma_{y}$ operation and three $\mathrm{Li}$

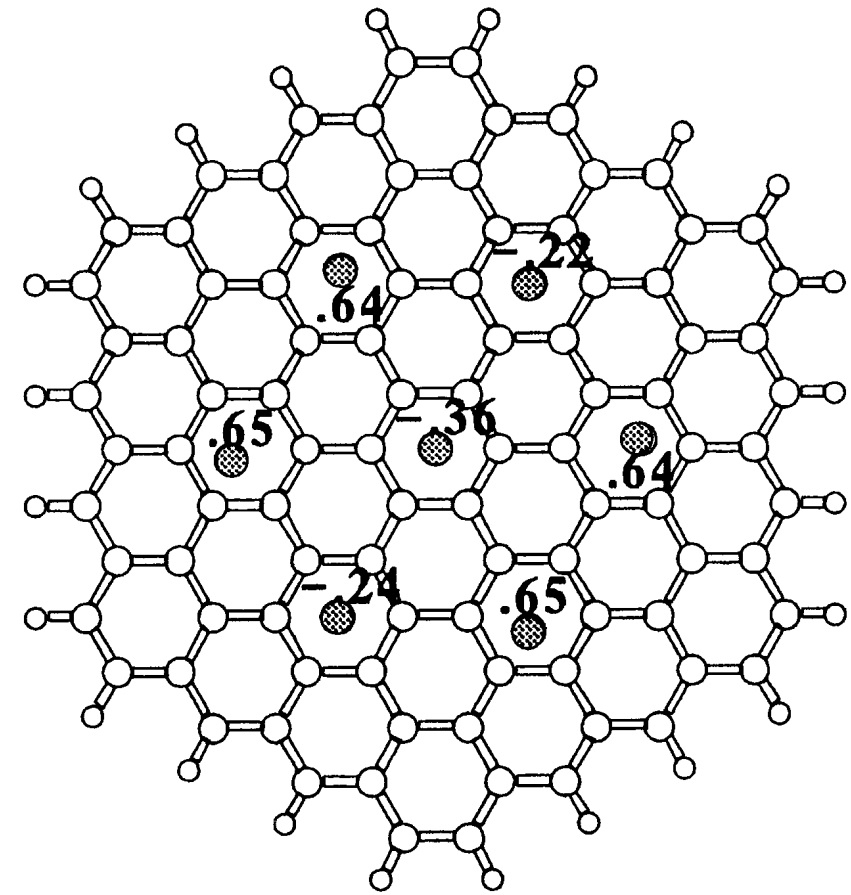

FIG. 7. An optimized Li geometry in the $\mathrm{Li}_{7} \mathrm{C}_{96} \mathrm{H}_{24}$ cluster. The $\mathrm{Li}$ ionicity can be negative in the case of a finite cluster (see text for details).

ions are located along the $y$-axis. The final geometry of the $\mathrm{Li}$ ions in Fig. 7 is not hexagonal, but rather has $C_{2}$ symmetry because of the Jahn-Teller effect, and thus all the $\mathrm{Li} 2 s$ orbitals are nondegenerate.

When we put five occupied electrons into MO's below the Fermi level, the ionicity is determined. Since the amplitude of the MO's for the three Li ions shown in Fig. 7 is large for the occupied MO's, these $\mathrm{Li}$ ions become negatively charged. It is thus not a numerical error that such a situation occurs for a small cluster as a kind of surface charge oscillation effect. When the number of $\mathrm{Li}$ ions increases, there are many occupied MO's with different symmetries and thus the probability for Li MO's to appear below the Fermi energy will quickly converge to a constant value. Thus, the inhomogeneous charge distribution of a $\mathrm{Li}$ ion on a graphite surface will be smeared out to yield the Li ionicity of a bulk GIC. It is thus concluded that the large broadening for the ${ }^{7} \mathrm{Li}$ NMR Knight shift spectra ${ }^{2}$ not only comes from the Li ions at the edges but also from the $\mathrm{Li}$ ions on the graphite cluster because of this finite size effect. It is noted that the symmetry-lowering effect is another important broadening mechanism for the ${ }^{7} \mathrm{Li}$ NMR Knight shift. ${ }^{2}$

\section{E. Partially hydrogen-terminated edges: $\mathrm{Li}_{7} \mathrm{C}_{96} \mathrm{H}_{20}$ and $\mathrm{Li}_{7} \mathrm{C}_{96} \mathrm{H}_{16}$ clusters}

Finally we mention the cases of partially hydrogenterminated edges: $\mathrm{Li}_{7} \mathrm{C}_{96} \mathrm{H}_{20}$ in which four $\mathrm{H}$ atoms at one edge of a hexagonal $\mathrm{C}_{96}$ cluster are removed, 
and $\mathrm{Li}_{7} \mathrm{C}_{96} \mathrm{H}_{16}$ in which eight $\mathrm{H}$ atoms at two edges are removed. We consider three cases for the relative positions of the two edges from which the $\mathrm{H}$ atoms are removed in $\mathrm{Li}_{7} \mathrm{C}_{96} \mathrm{H}_{16}$, depending on whether the two edges are in (1) nearest-neighbor, (2) next-nearestneighbor, or (3) opposite positions, which we call the ortho, meta, and para positions of $\mathrm{C}_{96} \mathrm{H}_{16}$, respectively, using terminology similar to that for hydrogen atoms of the benzene molecule $(\mathrm{CH})_{6}$.

In the $\mathrm{Li}_{7} \mathrm{C}_{96} \mathrm{H}_{20}$ cluster, seven $\mathrm{Li}$ ions are stable, as is observed in the $\mathrm{Li}_{7} \mathrm{C}_{96} \mathrm{H}_{24}$ cluster. The $\mathrm{Li}$ positions are, however, more distorted in the case of $\mathrm{Li}_{7} \mathrm{C}_{96} \mathrm{H}_{20}$ than for $\mathrm{Li}_{7} \mathrm{C}_{96} \mathrm{H}_{24}$, although the $\mathrm{Li}$ atoms in both cases are around the center of the hexagonal ring.

In the $\mathrm{Li}_{7} \mathrm{C}_{96} \mathrm{H}_{16}$ clusters, some $\mathrm{Li}$ ions move from their initial $\sqrt{3} \times \sqrt{3}$ structure, and one $\mathrm{Li}$ ion in the case of meta and para $\mathrm{Li}_{7} \mathrm{C}_{96} \mathrm{H}_{16}$ clusters shows $\sigma$ bonding to dangling bonds observed in Fig. 2. An interesting result is that $\mathrm{Li}_{2}$ or $\mathrm{Li}_{3}$ clusters are obtained on the graphite surface, in ortho and para $\mathrm{Li}_{7} \mathrm{C}_{96} \mathrm{H}_{16}$ clusters, respectively.

In the para $\mathrm{Li}_{7} \mathrm{C}_{96} \mathrm{H}_{16}$ cluster, one $\mathrm{Li}$ ion forms a $\sigma$ bond with a dangling carbon bond, two Li ions are ionic on the cluster, one $\mathrm{Li}$ ion is ionic at a hydrogenterminated edge, and the remaining three $\mathrm{Li}$ ions form a $\mathrm{Li}_{3}$ triangular cluster. The $\mathrm{Li}_{3}$ triangle cluster that we obtained is perpendicular to the graphene surface. One edge ( $\mathrm{Li}-\mathrm{Li}$ distance $2.50 \AA$ ) of the triangle is parallel to the graphite surface and the other two edges are longer $(3.03 \AA)$. Although there is charge transfer (totally $0.4 e$ ) from the $\mathrm{Li}_{3}$ triangular cluster to the graphite surface, the $\mathrm{Li}_{3}$ cluster forms a kind of molecular state related to that proposed by Sato et al. ${ }^{2}$

We also find one $\mathrm{Li}_{2}$ cluster in the optimized ortho $\mathrm{Li}_{7} \mathrm{C}_{96} \mathrm{H}_{16}$ cluster, where the remaining five $\mathrm{Li}$ ions are ionic on the graphite cluster. The two $\mathrm{Li}$ ions facing the nearest-neighbor edges are very much distorted, and thus form a $\mathrm{Li}_{2}$ cluster with a $\mathrm{Li}-\mathrm{Li}$ bond that is almost parallel to the graphene surface. The distances of the two $\mathrm{Li}$ ions from the graphene surface are $2.49 \AA$ and $2.47 \AA$, and the ionicities of the $\mathrm{Li}$ ions are $+0.30 e$ and $+0.21 e$, respectively, which are much smaller than the ionicity for ionic bonds. However, this ionicity should be relevant to Li MO orbitals, as discussed in the previous subsection. The $\mathrm{Li}-\mathrm{Li}$ distance of the $\mathrm{Li}_{2}$ cluster $(2.72 \AA)$ is smaller than the other $\mathrm{Li}-\mathrm{Li}$ average distance $(4.3 \AA)$ and close to the lattice constant $(2.46 \AA)$ of graphite. Thus, this situation is very close to what Sato et $a .^{2}$ proposed. However, the two Li positions are not over the center of the hexagonal ring but almost on $\mathrm{C}-\mathrm{C} \sigma$ bonds, when viewed from the top. Furthermore, the density of $\mathrm{Li}$ ions on the graphite plane is close to that in Li-GIC's. Thus it seems to be difficult to say at present that a molecular cluster is essential for excess Li ions.

\section{SUMMARY}

These calculated results show that there are two basic sites for $\mathrm{Li}$ ions in graphite clusters which form models for pregraphitic carbons or micrographites. Two different chemical bonds are associated with these sites, which have different ionicities, and can be identified with ionic $\left(\mathrm{Li}^{+0.6 e}\right)$ or covalent $\left(\mathrm{Li}^{+0.3 e}-\mathrm{Li}^{+0.5 e}\right)$ bonds. The different ionicities of the ionic or covalent bonds depend on (1) whether the edge is hydrogen terminated or not, (2) whether the Li ion is within the cluster or is bonded to dangling bonds at the edges, and (3) whether the $\mathrm{Li}$ ion at the edge forms a bond to one or to two carbon atoms, respectively. An important factor for giving different ionicities for different sites in the cluster is the finite size effect in which a finite number of occupied Li MO's with lower symmetry may result in an inhomogeneous charge distribution. Further, as is observed in a partially H-terminated system, $\mathrm{Li}_{2}$ or $\mathrm{Li}_{3}$ clusters could also give rise to an inhomogeneous charge distribution. Thus in the small cluster, there are several possible broadening mechanisms for NMR Knight shift spectra.

The identification of these two types of sites provides a possible reason why NMR Knight shift experiments basically show two charge transfer states, as is also observed in the present computer calculation. In the case of the $\mathrm{C}_{96}$ cluster considered in our model, when we put $\mathrm{Li}$ ions on all $18 \sigma$ bond sites as shown in Fig. 2, or on 6 ionic $A 1$ sites as in Fig. 5, and at ionic sites on both sides of the graphite surface in the $\sqrt{3} \times \sqrt{3}$ structure as is observed in $\mathrm{LiC}_{6}$ GIC's (corresponding, respectively, to $A, 6 E$, and $6 D$ sites of Fig. $1,13 \times 2=26$ ), we arrive at a total number of $50 \mathrm{Li}$ ions. The ratio of $\mathrm{Li}$ to $\mathrm{C}$ is thus close to 1 to 2 , and the ratio of covalent $\mathrm{Li}$ ions to ionic $\mathrm{Li}$ ions is 9 to 16 . In fact, we have obtained the result for up to $13 \mathrm{Li}$ atoms within the capacity of the computer. Since there are many more inequivalent $\mathrm{Li}$ sites for ionic $\mathrm{Li}$ ions compared with localized covalent Li ions, the NMR spectral line for the ionic Li should be broader than that for the covalent Li. These considerations seem to be consistent with the experimental results.

We can expect a dependence of NMR spectra on the size of the cluster. When the size of the cluster increases, the density of surface sites decreases relatively and the covalent contribution decreases. Since the area and the surface are proportional to $L^{2}$ and $L$, respectively, where $L$ is the size of the cluster, the ratio of covalent to ionic sites decreases as $1 / L$. This situation should become clarified from reversibility experiments on secondary $\mathrm{Li}$ batteries, since we expect the covalently bonded Li ions to be too stable for reversible charging and discharging. Further, the finite size effect of the ionicity of Li ions in hydrogen-terminated clusters should quickly decrease with increasing $L$, which can be seen in the NMR 
spectra, through a narrowing of the line width and a strengthening of the intensity.

Another possible experiment that should be sensitive to the size effect of clusters would be a study of hydrogen passivation of the dangling bonds of the cluster before $\mathrm{Li}$ intercalation. In this case there should be no covalently bonded states for Li ions and only the finite size effect associated with the ionicity of $\mathrm{Li}$ ions with ionic bonding should be observed in this case.

In summary, we have calculated the optimized geometry of $\mathrm{Li}$ ions on a graphite cluster. We obtain not only ionic $\mathrm{Li}$ states but also covalent $\mathrm{Li}$ states at the edge of the cluster. There are many possible Li doping sites, depending on the finite size of the cluster which will be relevant to the excess $\mathrm{Li}$ ions observed in small graphite clusters.

\section{ACKNOWLEDGMENTS}

The authors gratefully acknowledge useful discussions with Professor M. Endo and Professor T. Enoki, and with M.J. Matthews. The present collaborative research is funded in part by the International Joint Research Program of the New Energy and Industrial Technology Organization (NEDO), Japan. The authors thank the Supercomputer Center, Institute for Solid State Physics, University of Tokyo for the facilities and the use of the FACOM VPP500. Part of the work by RS is supported by a Grant-in Aid for Scientific Research (B) (No. 08454079) from the Ministry of Education, Science and Culture, of Japan. The MIT work was partly supported by the NSF (DMR 95-10093) and by the Lawrence Livermore National Laboratory through subcontract (No. B287707).

\section{REFERENCES}

1. M. Endo, K. Takeuchi, S. Igarashi, K. Kobori, M. Shiraishi, and H. W. Kroto, J. Phys. Chem. Solids 54, 1841 (1993).

2. K. Sato, M. Noguchi, A. Demachi, N. Oki, and M. Endo, Science 264, 556 (1994).

3. K. Tanaka, S. Yata, and T. Yamabe, Synthetic Metals 71, 2147 (1995).

4. S. Yata, Y. Hato, H. Kinoshita, N. Ando, A. Anekawa, T. Hashimoto, M. Yamaguchi, K. Tanaka, and T. Yamabe, Synthetic Metals 73, 273 (1995).

5. S. Yata, H. Kinoshita, M. Komori, N. Ando, T. Kashiwamura, T. Harada, K. Tanaka, and T. Yamabe, Synthetic Metals 62, 153 (1994).

6. M. S. Dresselhaus and G. Dresselhaus, Adv. Phys. 30, 139-326 (1981).

7. H. W. Kroto, J. R. Heath, S.C. O'Brien, R.F. Curl, and R.E. Smalley, Nature (London) 318, 162-163 (1985).

8. M.S. Dresselhaus, G. Dresselhaus, and P.C. Eklund, Science of Fullerenes and Carbon Nanotubes (Academic Press, San Diego, CA, 1996).

9. T.P. Martin, N. Malinowski, U. Zimmermann, U. Näher, and H. Schaber, J. Chem. Phys. 99, 4210 (1993).

10. C. Kittel, in Introduction to Solid State Physics, 6th ed. (John Wiley and Sons, New York, 1986).

11. J. J.P. Stewart, Fujitsu Limited, Tokyo, Japan (1993); semiempirical quantum chemistry library.

12. J. J. P. Stewart, J. Comput. Chem. 10, 209 (1989).

13. J. J.P. Stewart and M. B. Coolidge, J. Comp. Chem. 12, 1157 (1991).

14. M. J. Matthews, M. S. Dresselhaus, M. Endo, Y. Sasabe, T. Takahashi, and K. Takeuchi, J. Mater. Res. 11, 3099 (1996).

15. R. Saito, M. Fujita, G. Dresselhaus, and M. S. Dresselhaus, Phys. Rev. B 46, 1804 (1992).

16. M. Fujita, K. Wakabayayashi, K. Nakada, and K. Kusakabe, J. Phys. Soc. Jpn. 65, 1920 (1996).

17. M. Fujita, M. Yoshida, and K. Nakada, Fullerene Sci. Technol. 4, 565 (1996).

18. K. Nakada, M. Fujita, G. Dresselhaus, and M. S. Dresselhaus, Phys. Rev. B 54, 17954 (1996). 